\title{
Stress analysis of metallic thick-walled high-pressure elbows overwrapped with composite material
}

\author{
Yong Xiao ${ }^{1,2}$, Yefa $\mathrm{Hu}^{1,2}$, Jinguang Zhang ${ }^{1,2,}{ }^{*}$, Chunsheng Song ${ }^{1,2}$, Xiangyang Huang ${ }^{1,3}$, and Zhaobing Liu ${ }^{1,2}$ \\ ${ }^{1}$ School of Mechanical and Electronic Engineering, Wuhan University of Technology, Luoshi Road 122, Wuhan 430070, Hubei, \\ PR China \\ ${ }^{2}$ Institute of Advanced Materials Manufacturing Equipment and Technology, Wuhan University of Technology, Luoshi Road 122, \\ Wuhan 430070, Hubei, PR China \\ ${ }^{3}$ Hishan Machinery Co., Ltd., Hishan Road 666, Putian 351100, Fujian, PR China
}

Received: 30 September 2017 / Accepted: 26 February 2018

\begin{abstract}
In this paper, Carbon fibre-reinforced plastic (CFRP) is used to reinforce metal elbow, which is a new concept and has the potential to improve the strength of metal elbow. For the elbow, the circumferential stress is the main factor for its failure. In this study, a new stress model of thick-walled high pressure elbow reinforced by composite material is presented to predict the stress distribution. Three-dimensional solid model of elbow is constructed and finite element simulations for the elbow are performed to verify the accuracy of the theoretical model. From the results obtained, the maximum circumferential stress of elbow being reinforced by CFRP is smaller than that of elbow not being reinforced by CFRP. The thinner the wall thickness of metal elbow, the more obvious the effect of CFRP will be. The thicker the wall thickness of metal elbow and the thinner the wall thickness of CFRP, the better the accuracy of stress model will be. When the wall thickness of metal elbow is $25 \mathrm{~mm}$, the deviation is smaller than $4 \%$. Therefore, the new stress model is suited for providing stress expression generally. In addition, failure analysis on metal elbow reinforced by CFRP shows that failure of metal layer is the major cause for failure of CFRP layer, i.e. if the metal layer do not fail, neither do CFRP layer. This provides more proof to justify the accuracy and application of the stress model considering the effect of CFRP.
\end{abstract}

Keywords: High pressure elbow / carbon fiber-reinforced plastic / stress analysis / finite element analysis

\section{Introduction}

The elbow, as a joint part of tubes, is a necessary pipefitting in composite products [1]. In the process of shale gas exploitation, the fracturing truck can bring a lot of mud after long-time working, and the metal elbow is subject to the erosion of high pressure flow medium. Tube corrosion will become more and more severe with the increasing of operation time, and the corrosion defects can cause wall to become thinner, pressure resistant ability to drop and even result in local corrosion body perforating or rupture accidents. And the thick-walled and heavy-weighted metal elbow will inevitably cause trouble for installation and transportation.

Considering this, new materials are required to replace or partially replace the metal to improve properties of the elbow and as a response, advanced composite material rise to be a potential material [2]. Carbon fibre reinforced

\footnotetext{
* e-mail: jgzhang@whut.edu.cn
}

plastic (CFRP) is an extremely strong and light fibre reinforced plastic which contains carbon fibres and epoxy resin. It has unique advantages over traditional materials, such as low density, high specific strength, specific stiffness and long fatigue life. And by application of metal elbows wrapped by CFRP, the above problems will no longer be a nuisance. The utility model relates to metal elbow wrapped by CFRP, which can reduce weight of the elbow, effectively improve the strength and prolong the service life. The binding of the outer layers of CFRP eliminates risk of flying debris caused by elbow bursting. Considering that it can use continuous fibre over the whole component area, provide superior strength-to-weight ratios together with simplicity of implementation, filament winding has become one of the most important and cost-effective production techniques for composites [3,4].

Due to the excellent characteristics of CFRP, it has been widely used in areas of aerospace, wind power, shipbuilding and automotive parts. Moreover, owing to needs of weight reduction and body strengthening, interest in pipe parts repaired or reinforced by composite materials has been rising. Meanwhile, many advanced methods have 


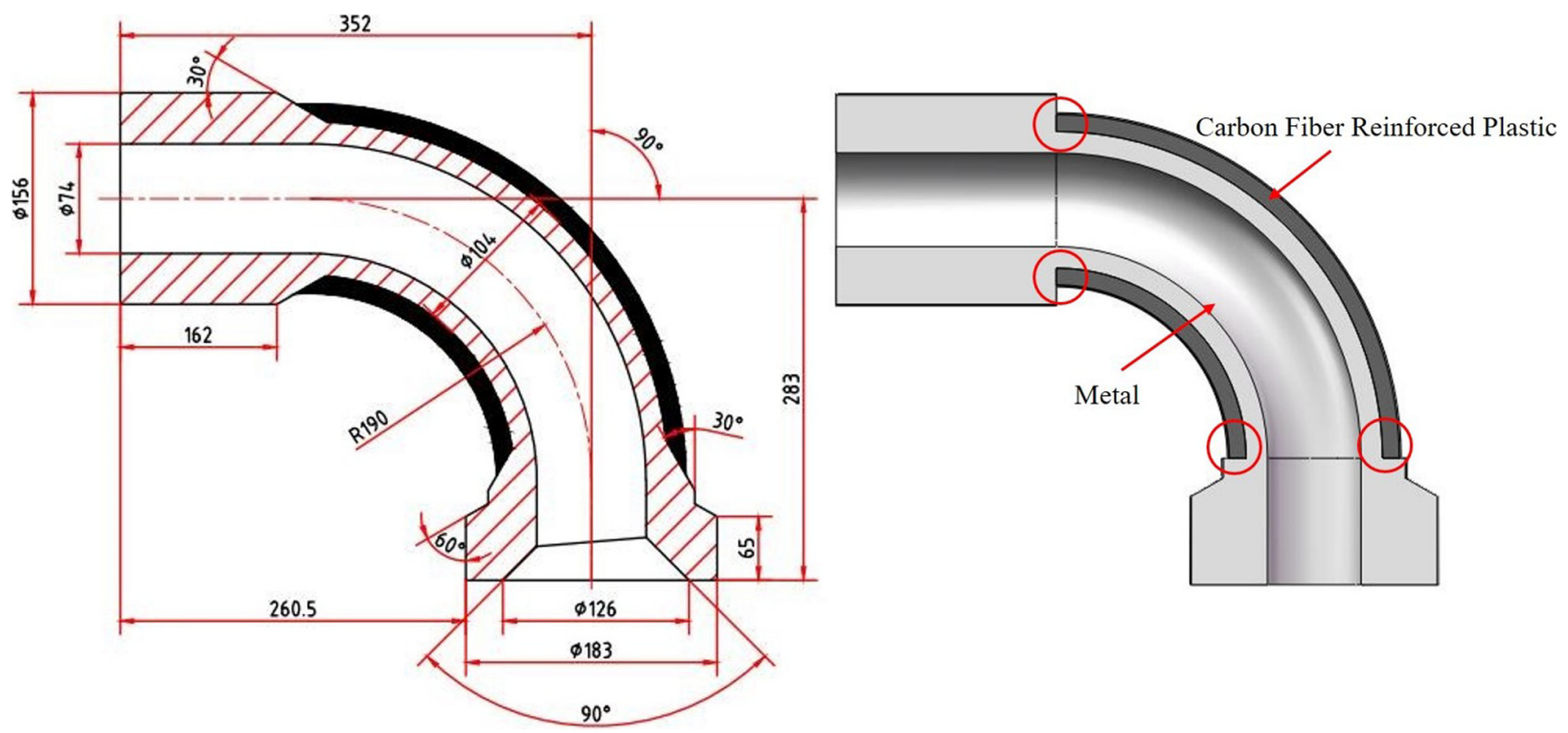

Fig. 1. Main dimensions and CAD model of metal elbow reinforced by CFRP (unit: mm).

been studied by several studies for improving the strength. Houssam Toutanji et al. [2] discussed the benefits of using CFRP and provided stress expressions on the interaction between different stresses exerted on pipe walls and the effects of CFRP sheets on the circumferential stresses of damaged pipe walls. Duell et al. [5] developed a new method to stop external corrosion and structurally reinforce steel pipes by externally wrapping damaged sections with fibre reinforced polymer materials. Gong et al. [6] presented a computational approach for safety assessment of submerged pipelines. Meniconi et al. [7] processed laboratory tests carried out to compare the behavior of fibre reinforced composite repairs applied to defects machined in pipeline test specimens. FANG et al. [8] reviewed the current understanding of the mechanism of stress corrosion cracking of pipeline steels. Badri et al. [9] demonstrated that composite pipeline showed higher stability than steel pipe. Altenbach et al. [10] studied the formation of stress field in a pipeline with a repair composite band during the solidification of the polymer resin. Pinheiro et al. [11] refined a methodology for fatigue life assessment of dented steel pipelines under cyclic internal pressure. Xie et al. [12] studied a new method to estimate the stress intensity factor based on the concept of the crack surface widening energy release rate. Wang et al. [13] recognized the influence of carbon on stress corrosion cracking of pipeline steels.

The pipe parts reinforced by composite material have been widely studied. Although some studies on stress state of straight pipe reinforced by composite material subject to pressure can be found, the stress state of elbow is very different from that of the straight pipe. Stress analysis which is used to improve strength of the elbow reinforced by composite material has never been investigated. The circumferential stress is the main factor for the failure of elbow, so it is important to get stress distribution. Therefore, based on analyses, the thick-walled high pressure elbow reinforced by composite material with new stress model is suggested in this study.

\section{Problem definitions}

\subsection{The CAD model of high pressure elbow}

Main dimension and CAD model of metal elbow reinforced by CFRP are shown in Figure 1. For the convenience of finite element analysis (FEA), parts marked by circles in Figure 1 are simplified. The total wall thickness of the elbow is between $12 \mathrm{~mm}$ and $33 \mathrm{~mm}$. According to the FEA, when the thickness of the metal elbow is $12 \mathrm{~mm}$, the maximum circumferential stress of the metal elbow is close to the maximum yield strength of the metal elbow. In this case, the metal elbow will fail. Therefore, the minimum wall thickness of the metal elbow is $12 \mathrm{~mm}$. When the thickness of the metal elbow is $33 \mathrm{~mm}$, the strength of the metal elbow is quite large.

\subsection{The material property of metal elbow reinforced by CFRP}

For the carbon/epoxy composite laminate, each ply has orthotropic material property and fibre direction of each ply can be different in practical applications. The part wrapping around metal elbow is full carbon fibre structure and made of M40J/Epoxy resin. See Table 1 for material properties of M40J/Epoxy resin. Nine material constants in Table 1 would be used in the FEA.

The metal elbow has isotropic material property and is made of 20CrNiMo. Table 2 lists material properties of 20CrNiMo. 
Table 1. The material properties of CFRP M40J/Epoxy resin.

\begin{tabular}{lllllllll}
\hline$E_{1}(\mathrm{GPa})$ & $E_{2}(\mathrm{GPa})$ & $E_{3}(\mathrm{GPa})$ & $v_{21}$ & $v_{32}$ & $v_{31}$ & $G_{12}(\mathrm{GPa})$ & $G_{23}(\mathrm{GPa})$ & $G_{13}(\mathrm{GPa})$ \\
\hline 231.1 & 8.16 & 8.16 & 0.3 & 0.3 & 0.42 & 3.19 & 3.19 & 2.88 \\
\hline
\end{tabular}

$E_{1}$ : longitudinal modulus, $E_{2}, E_{3}$ : transverse modulus; $v_{21}$ : 21-direction Poisson's ratio, $v_{31}$ : 31-direction Poisson's ratio, $v_{32}: 32-$ direction Poisson's ratio; $G_{12}, G_{13}$ : 12-direction, 13-direction shear modulus, $G_{23}$ : 23-direction shear modulus.

Notes: The material properties in Table 1 come from the Testing Center of Lianyungang Shenying Carbon Bike Co., Ltd.

Table 2. The material properties of $20 \mathrm{CrNiMo}$.

\begin{tabular}{llll}
\hline$E(\mathrm{~Pa})$ & $v$ & $\rho\left(\mathrm{kg} / \mathrm{m}^{3}\right)$ & $\sigma s(\mathrm{~Pa})$ \\
\hline $2.08 \mathrm{E}+11$ & 0.295 & $7.87 \mathrm{E}+03$ & $7.85 \mathrm{E}+08$ \\
\hline
\end{tabular}

$E$ : elastic modulus, $v$ : Poisson's ratio, $\rho$ : density, $\sigma s$ : yield strength.

Notes: The material properties in Table 2 come from the Sinopec Petroleum Engineering Machinery Co., Ltd. fourth Machinery Plant.

\subsection{Determination of lay-up scheme}

In this study, the thickness of elbow is the only variable, while fibre direction and stacking sequence are of the same. The stacking sequence is only selected from fibre directions, i.e. $90^{\circ}$. Axis direction along the elbow is the reference direction of the fibre angle. The thickness of each ply is $0.075 \mathrm{~mm}$.

\section{Methods of analyses}

\subsection{Theoretical model of stress}

\subsubsection{Stress model of thick walled straight tube subjected} to internal pressure

The thick walled straight tube subjected to internal pressure is widely used in the Engineering. When the ratio of the outer radius $b$ to the inner radius $a$ of the straight tube is greater than 1.2 , it is called thick walled straight tube. Assuming that the thick walled straight tube is subjected to uniform internal pressure $P$. The stress state of the straight tube subjected to internal pressure is shown in Figure 2 under the condition of plane strain. The inner radius is $a$, the outer radius is $b$, the length is $L$ [14].

Cylindrical coordinate $(r, \theta, z)$ is taken, $z$ axis coincides with cylinder axis. The radial displacement along the $r$ direction is $u$. Because of the axisymmetric problem, the circumferential displacement $v=0$. Under the condition of plane strain, $\varepsilon_{z}=0$, therefore, the geometric relationships are as follows:

$$
\varepsilon_{r}=\frac{(u+d u)-u}{d r}=\frac{d u}{d r}, \quad \varepsilon_{\theta}=\frac{(r+u) d \theta-r d \theta}{r d \theta}=\frac{u}{r} .
$$

Deformation compatibility equation is as follow:

$$
\frac{d \varepsilon_{\theta}}{d r}=\frac{1}{r}\left(\varepsilon_{r}-\varepsilon_{\theta}\right)
$$

Physical equations are as follows:

$$
\begin{gathered}
\varepsilon_{r}=\frac{1}{E}\left[\sigma_{r}-\mu\left(\sigma_{\theta}+\sigma_{z}\right)\right], \\
\varepsilon_{\theta}=\frac{1}{E}\left[\sigma_{\theta}-\mu\left(\sigma_{r}+\sigma_{z}\right)\right] .
\end{gathered}
$$

The equilibrium equation under axisymmetric condition is as follow:

$$
\begin{gathered}
\left(\sigma_{r}+d \sigma_{r}\right)(r+d r) d \theta-\sigma_{r} r d \theta-2 \sigma_{\theta} d r \sin \frac{\theta}{2}=0, \\
\frac{d \sigma_{r}}{d r}+\frac{\sigma_{r}-\sigma_{\theta}}{r}=0 .
\end{gathered}
$$

According to $(1) \sim(5)$, the following equation can be obtained:

$$
r \frac{d^{2} \sigma_{r}}{d r^{2}}+\frac{d \sigma_{r}}{d r}=0 .
$$

The boundary conditions of stress are as follows:

$$
\left.\begin{array}{l}
\sigma_{r 1}=-P(r=a) \\
\sigma_{r 1}=0(r=b)
\end{array}\right\} .
$$

It is assumed that there is no plastic deformation for thick walled cylinder subjected to internal pressure, always in the elastic stage, the solutions of equation (6) are as follow:

$$
\begin{gathered}
\sigma_{r 1}=\frac{P a^{2}}{b^{2}-a^{2}}\left(1-\frac{b^{2}}{r^{2}}\right), \\
\sigma_{\theta 1}=\frac{P a^{2}}{b^{2}-a^{2}}\left(1+\frac{b^{2}}{r^{2}}\right), \\
\sigma_{z 1}=\frac{P a^{2}}{b^{2}-a^{2}} .
\end{gathered}
$$

$\sigma_{r 1}$ : radial stress of straight tube, $\sigma_{\theta 1}$ : circumferential stress of straight tube, $\sigma_{z 1}$ : axial stress of straight tube.

For thick walled straight tube, the maximum circumferential stress is located at the inner wall, in this case $r=a$, the maximum circumferential stress is:

$$
\sigma_{\theta 1 \max }=\frac{P a^{2}}{b^{2}-a^{2}}\left(1+\frac{b^{2}}{a^{2}}\right) .
$$



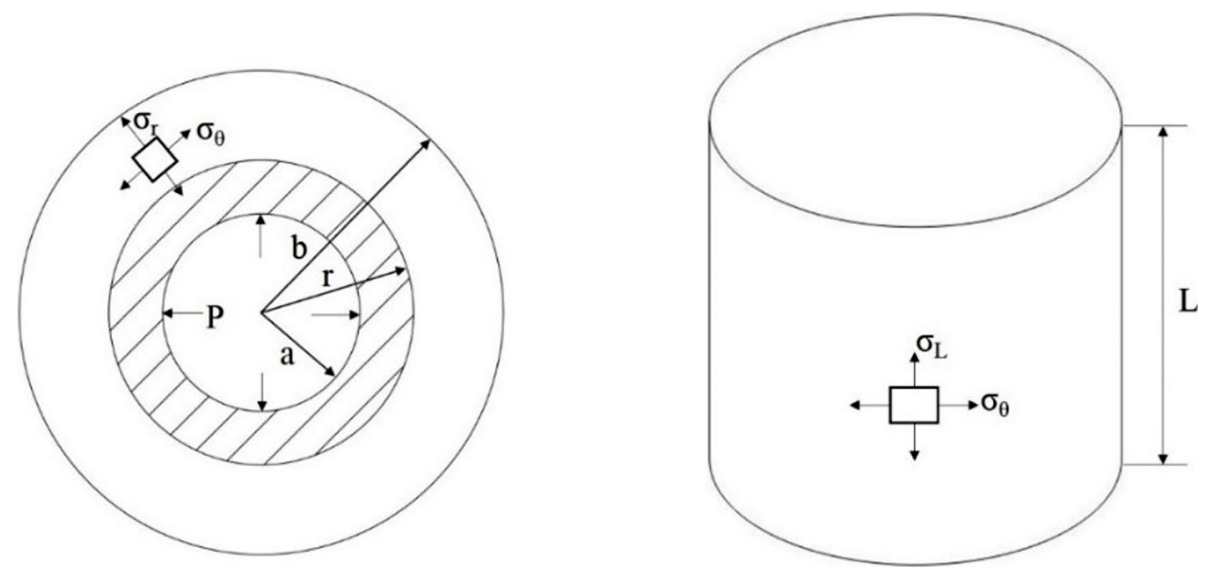

Fig. 2. Cross section of thick walled tube under internal pressure. tube.

$\sigma_{\theta 1 \mathrm{max}}:$ maximum circumferential stress of straight

\subsubsection{Stress model of metal elbow subjected to internal pressure}

In the process of bending, the outer wall of the elbow is thinner because of the stretching, the inner wall is thickened due to compression, the elliptical effect occurs in cross section at the same time, therefore, the stress state of elbow is more complicated than that of straight tube under internal pressure. For stress analysis of elbow subjected to internal pressure, the elbow can no longer be regarded as a long and closed cylindrical vessel, it can only be seen as a plane curve around an axis of rotation to generate toroidal shell, the internal pressure acting on the toroidal shell is axisymmetric load. There is no doubt that the stress distribution along the wall of tube is different from that of straight tube. Under the effect of internal pressure, the circumferential stress in the same layer is unequal, the outside is the smallest, the inner is the largest.

The circumferential stress is the main factor for the failure of elbow, the variation of the circumferential stress of the elbow compared with straight tube can be expressed as follow [15]:

$$
\sigma_{\theta}=\sigma_{\theta 1} \frac{2 R+a \sin \alpha}{2(R+a \sin \alpha)} .
$$

In this equation, $\sigma_{\theta 1}$ is the circumferential stress of the straight tube, Comparison of elbow and straight tube, the expression $\frac{2 R+r \sin \alpha}{2(R+r \sin \alpha)}$ is the coefficient of variation, it is called stress intensity coefficient.

The distribution of circumferential stress on the crosssection of the elbow is unequal. According to the equation (12), it is concluded that the maximum circumferential stress occurs in the inner surface of the elbow. Here, $\alpha=270^{\circ}, r=a$. Therefore, $\sin \alpha=-1$.

$$
\begin{aligned}
\sigma_{\max } & =\sigma_{\theta 1 \max } \frac{2 R-r}{2(R-r)} \\
& =\frac{P a^{2}}{b^{2}-a^{2}}\left(1+\frac{b^{2}}{a^{2}}\right) \frac{2 R-a}{2(R-a)} .
\end{aligned}
$$

$\sigma_{\max }:$ maximum circumferential stress of metal elbow.

\subsubsection{Stress model of metal elbow reinforced by composite} material

Based on the equivalent circumferential stress at the interface between pipe wall and fiber reinforcement layer, the thickness of the CFRP layer is converted to the thickness of the metal tube. The following equation can be obtained:

$$
t_{s}^{\prime}=\frac{E_{c} t_{c}}{E_{s}}
$$

$t_{s}^{\prime}$ : Equivalent thickness of metal layer; $E_{\mathrm{c}}$ : Elastic modulus of fiber reinforced layer in the main direction; $E_{\mathrm{s}}$ : elastic modulus of metal tube; $t_{c}$ : thickness of fiber reinforced layer.

The equivalent outer radius $b$ is as follow:

$$
b^{\prime}=a+t_{s}+\frac{E_{c} t_{c}}{E_{s}} .
$$

According to Sections 3.1.1 and 3.1.2, the maximum circumferential stress of metal elbow reinforced by composite material also occurs in the inner surface of the elbow. Here, $\alpha=270^{\circ}, r=a$. Therefore, $\sin \alpha=-1$.

$$
\begin{aligned}
\sigma_{\max } & =\sigma_{\theta 1 \max }{ }^{\prime} \frac{2 R-r}{2(R-r)} \\
& =\frac{P a^{2}}{b^{\prime 2}-a^{2}}\left(1+\frac{b^{\prime 2}}{a^{2}}\right) \frac{2 R-a}{2(R-a)},
\end{aligned}
$$

$\sigma_{\theta 1 \max }$ : maximum circumferential stress of equivalent straight tube; $\sigma_{\max }$ : maximum circumferential stress of metal elbow reinforced by composite material.

\subsection{Finite element models}

In this study, take metal elbow whose wall thickness is $15 \mathrm{~mm}$ as an example, being reinforced by CFRP whose wall thickness is $6 \mathrm{~mm}$. A FEA is performed including model and mesh, load, boundary condition, solving, and 


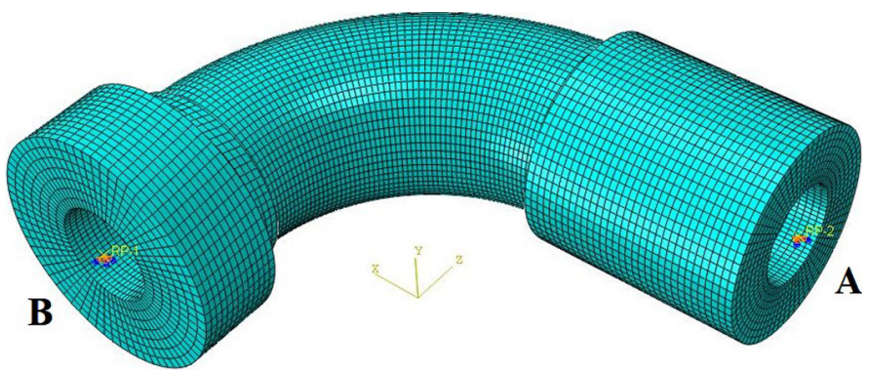

Fig. 3. Finite element model for high pressure elbow.

post-processing. Figure 3 shows the finite element model of high pressure elbow. By defining density, elasticity modulus and Poisson's ratio to the metal elbow, the metal elbow is modelled by SOLID C3D8R elements, the number of elements is 4422 and the approximate global size is $5 \mathrm{~mm}$. After giving it solid and homogeneous section properties, structure method is used to divide the hexahedral mesh. By defining properties of engineering constant and failure parameters for CFRP layers, the CFRP layer is modelled by the continuum shell SC8R elements with 30-ply settings, and structure method is also used to divide the hexahedral mesh, the number of elements is 40186 and the approximate global size is $5 \mathrm{~mm}$ as well. Layers are set up in the composite layup manager through discrete coordinate system method and stack direction is selected from the inner wall to the outer wall along the thickness direction. Layup direction is $90^{\circ}$ and thickness of a single layer is $0.2 \mathrm{~mm}$.

Loading and boundary conditions are also shown in Figure 3. As shown in Figure 3, the boundary conditions are prescribed on the specified nodes of the finite element model based on working condition of high pressure elbow. At End A of the tube, the elbow can rotate around the axis direction of End A, while other degrees of freedom of End A are constrained to zero. Meanwhile, the other end (End B) of the elbow are fixed in all directions. For the solid elements, the fixed constraints on the nodes are zero displacement and zero rotation. The internal pressure value of the elbow is $175 \mathrm{MPa}$.

\subsection{Failure criterion}

For the failure of metal layer of elbow, whether the maximum circumferential stress carried by it is higher than the yield strength of the material is our main criterion. However, due to the anisotropy of composite, failure of CFRP layer can be harder to determine. Since the maximum stress criterion and the maximum strain criterion have been proven unsatisfactory in predicting failure behavior of composite, several failure criteria that are more scientific and precise have been developed. According to Tsai-Wu theory, under plane stress, polynomial criterion for orthotropic single lamina is a relatively complete criterion to determine whether the single lamina of composite fails. This theory was developed into the 3D Tsai-Wu Criterion later on and was used for predicting failure of parts of axial and laminated composites that can not be predicted under simple plane stress.
Tsai-Wu Criterion is used for failure analysis on parts of carbon fibre tube and the expression is indicated as follows:

$$
\begin{gathered}
F I=F_{1} \sigma_{1}+F_{2} \sigma_{2}+F_{3} \sigma_{3}+F_{11} \sigma_{1}^{2}+F_{22} \sigma_{2}^{2}+F_{33} \sigma_{3}^{2} \\
+F_{44} \sigma_{4}^{2}+F_{55} \sigma_{5}^{2}+F_{66} \sigma_{6}^{2}+2 F_{12} \sigma_{1} \sigma_{2} \\
+2 F_{13} \sigma_{1} \sigma_{3}+2 F_{23} \sigma_{2} \sigma_{3} \geq 1
\end{gathered}
$$

where

$$
\left.\begin{array}{l}
F_{1}=\frac{1}{S_{1}^{t}}+\frac{1}{S_{1}^{c}}, F_{2}=\frac{1}{S_{2}^{t}}+\frac{1}{S_{2}^{c}}, F_{3}=\frac{1}{S_{3}^{t}}+\frac{1}{S_{3}^{c}} \\
F_{11}=-\frac{1}{S_{1}^{t} S_{1}^{c}}, F_{22}=-\frac{1}{S_{2}^{t} S_{2}^{c}}, F_{33}=-\frac{1}{S_{3}^{t} S_{3}^{c}} \\
F_{44}=\frac{1}{S_{4}^{2}}, F_{55}=\frac{1}{S_{5}^{2}}, F_{66}=\frac{1}{S_{6}^{2}} \\
F_{12}=-\frac{\sqrt{F_{11} F_{22}}}{2}, F_{13}=-\frac{\sqrt{F_{11} F_{33}}}{2}, F_{23}=-\frac{\sqrt{F_{22} F_{33}}}{2}
\end{array}\right\} .
$$

FI: Tsai-Wu failure index and when it was higher than 1 , it indicated that material was failed; $\sigma_{1}, \sigma_{2}, \sigma_{3}, \sigma_{4}, \sigma_{5}, \sigma_{6}$ : the normal stress along 1,2 and 3 directions and shear stress on planes $2-3,1-3$ and $1-2 ; S_{1}^{t}, S_{1}^{c}, S_{2}^{t}, S_{2}^{c}, S_{3}^{t}, S_{3}^{c}$ : tensile strength and compression strength along directions of materials 1,2 and $3 ; S_{4}, S_{5}, S_{6}$ : the shear stress on planes 2-3, 1-3 and 1-2.

\subsection{Manufacturing procedure of high elbow}

Single direction prepreg belt (the width is $10 \mathrm{~mm}$ ) is wrapped manually, due to technological limitations, $45^{\circ}$ direction of winding can not be used manually, so the approximate direction of $90^{\circ}$ winding is adopted. The prepreg belt is kept straight and twisted from one end to the other, winding the elbow until the outer diameter is about $152 \mathrm{~mm}$. Then, a high temperature film is wrapped outside the elbow and fixed with a high-temperature textured adhesive tape. The vacuum bag is evacuated and then cured at high temperature. The manufacturing procedure is shown in Figure 4.

\section{Results and discussion}

In this study, the results calculated by analytical analysis and FEA are presented to study the new stress model.

\subsection{Finite element results}

FEA is used to verify the accuracy of the theoretical model. Both metal elbows with different wall thickness that is and is not being reinforced by CFRP are analyzed.

Take metal elbow with $15 \mathrm{~mm}$ wall thickness as an example, not being reinforced by CFRP, where the circumferential stress distribution is shown in Figure 5. As shown in Figure 5, under effect of internal pressure, 


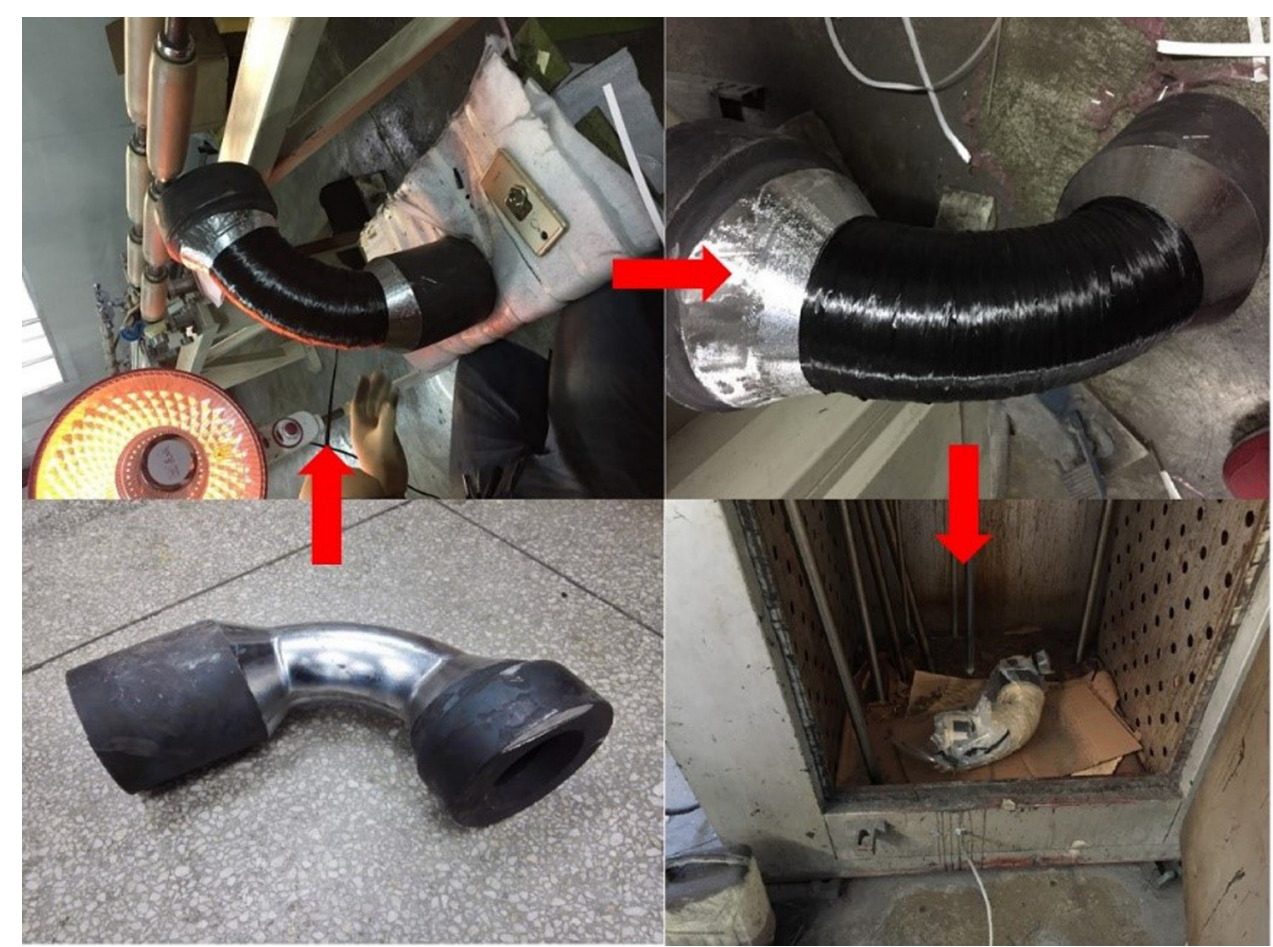

Fig. 4. Manufacturing procedure of high elbow.

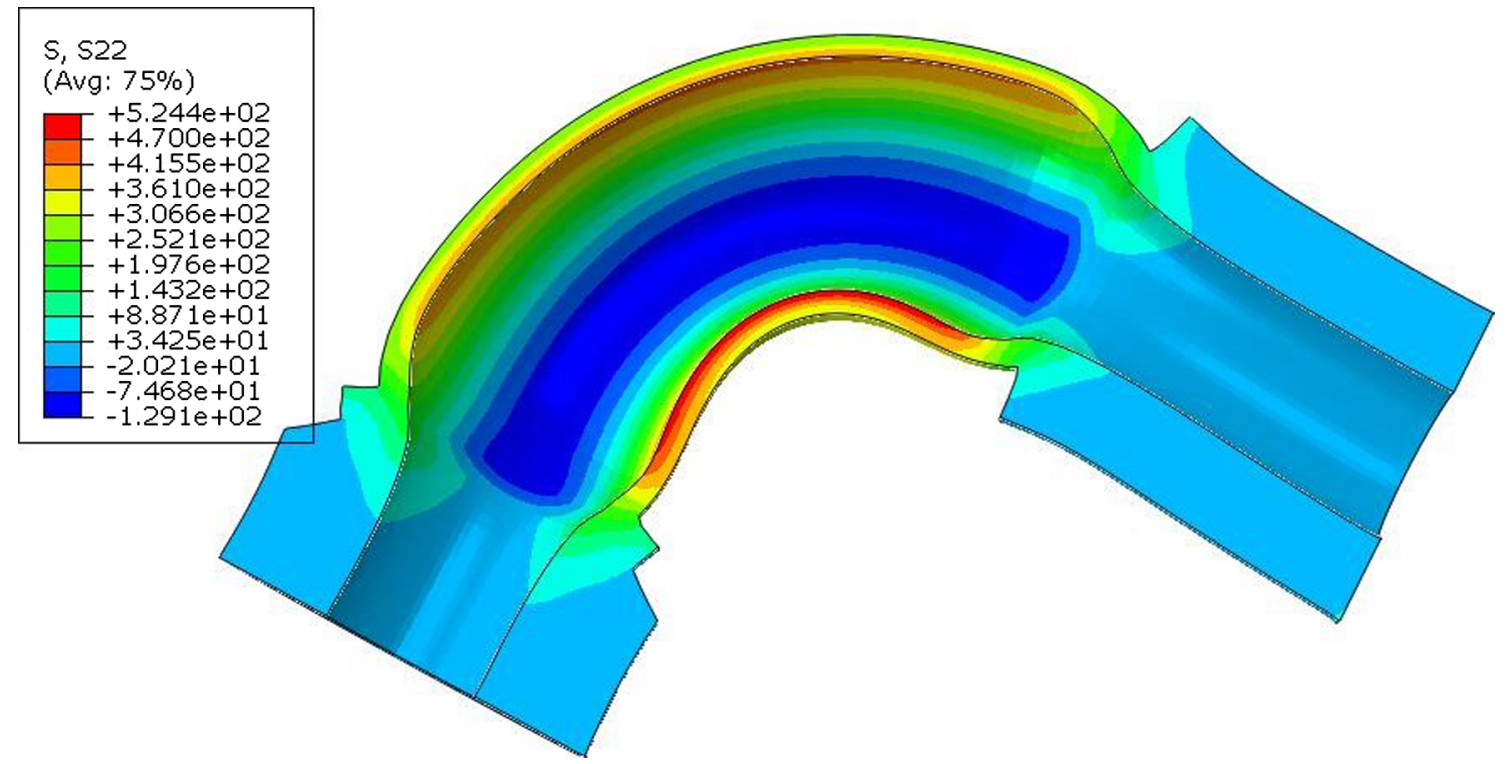

Fig. 5. Circumferential stress distribution of metal elbow (the wall thickness is $15 \mathrm{~mm}$ ).

circumferential stress on the same layer is unequal: the outer have the smallest while the inner had the largest; the maximum circumferential stress occurs on the inner surface of the elbow and is valued at $524.4 \mathrm{MPa}$.

Still take metal elbow whose wall thickness is $15 \mathrm{~mm}$ as an example, being reinforced by CFRP whose wall thickness is $6 \mathrm{~mm}$, and the circumferential stress distribution of metal elbow is shown in Figure 6. As shown in Figure 6, under the effect of CFRP, the maximum circumferential stress is less than that of the elbow not being reinforced by CFRP. The maximum circumferential stress also occurs on the inner surface of the elbow and is valued at $482.1 \mathrm{MPa}$.

Whether the maximum circumferential stress carried by the metal layer of elbow is higher than the yield strength of the material is our main criterion for failure of metal layer of elbow. And in terms of failure of CFRP layer, we'll use 3D Tsai-Wu Criterion. Due to the limitation of the size 


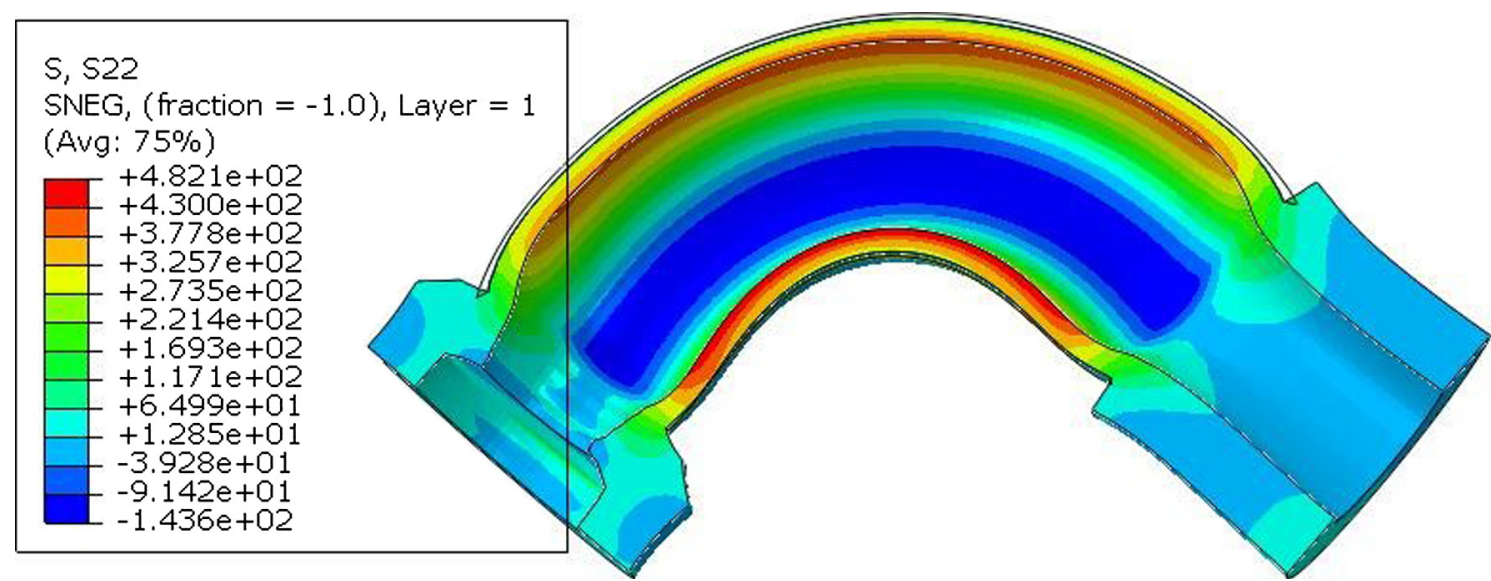

Fig. 6. Circumferential stress distribution of metal elbow reinforced by CFRP. (The wall thickness of elbow and CFRP is 15 and $6 \mathrm{~mm}$, respectively).

of the picture, Tsai-Wu failure indexes for three plies are selected. See Figure 7 for the calculation results.

\subsection{Comparison of theoretical analysis results and finite element results}

\subsubsection{Different wall thickness of metal elbow}

The wall thickness of the elbow is $12 \mathrm{~mm}, 15 \mathrm{~mm}$ and $24 \mathrm{~mm}$ respectively. Through theoretical calculation and FEA, the maximum circumferential stress of the elbow is shown in Figure 8.

As shown in Figure 8, for the metal elbow, the maximum circumferential stress is increasing as the wall thickness decreases. The maximum circumferential stress calculated by theoretical model is smaller than that calculated by FEA. The deviation between theoretical calculation and FEA is $4.7-5.4 \%$. When the wall thickness is $12 \mathrm{~mm}$, the average value of the maximum circumferential stress is $734.2 \mathrm{MPa}$. It exceeds the value of yield strength (the yield strength of elbow material is $785 \mathrm{Mpa}$ ), and the metal elbow is damaged.

\subsubsection{Metal elbow reinforced by CFRP with different wall thickness}

(1) Metal elbow with $25 \mathrm{~mm}$ wall thickness

When the wall thickness of metal elbow is $25 \mathrm{~mm}$, the wall thicknesses of CFRP reinforcing metal elbow are $6 \mathrm{~mm}$ and $12 \mathrm{~mm}$ respectively, Through theoretical calculation and FEA, the maximum circumferential stress of the elbow is shown in Figure 9.

As shown in Figure 9, when the wall thickness of CFRP is $6 \mathrm{~mm}$, the maximum circumferential stress decreases by $13.4 \%$ averagely. When the wall thickness of CFRP is $12 \mathrm{~mm}$, the maximum circumferential stress decreases by $21 \%$ averagely.

(2) Metal elbow with $15 \mathrm{~mm}$ wall thickness

When the wall thickness of metal elbow is $15 \mathrm{~mm}$, the wall thicknesses of CFRP reinforcing metal elbow are $6 \mathrm{~mm}$ and $12 \mathrm{~mm}$ respectively, through theoretical calculation and FEA, the maximum circumferential stress of the elbow is shown in Figure 10.

As shown in Figure 10, when the wall thickness of CFRP is $6 \mathrm{~mm}$, the maximum circumferential stress decreases by $8.3 \%$ averagely. When the wall thickness of CFRP is $12 \mathrm{~mm}$, the maximum circumferential stress decreases by $22 \%$ averagely.

(3) Metal elbow with $9 \mathrm{~mm}$ wall thickness

When the wall thickness of metal elbow is $9 \mathrm{~mm}$, the wall thicknesses of CFRP reinforcing metal elbow are $6 \mathrm{~mm}$ and $12 \mathrm{~mm}$ respectively, through theoretical calculation and FEA, the maximum circumferential stress of the elbow is shown in Figure 11.

As shown in Figure 11, when the wall thickness of CFRP is $6 \mathrm{~mm}$, the maximum circumferential stress decreases by $35.7 \%$ averagely. When the wall thickness of CFRP is $12 \mathrm{~mm}$, the maximum circumferential stress decreases by $48.1 \%$ averagely. From the above analysis, when the wall thickness of metal elbow and CFRP is $9 \mathrm{~mm}$ and $12 \mathrm{~mm}$ respectively, the effect of composite material on circumferential stress of thick-walled elbow subject to high pressure is very obvious.

\subsubsection{The deviation between theoretical calculation and FEA}

In order to verify the accuracy and scope of application of the stress model, it is needed to be compared with finite element results. Table 3 lists the deviations of 12 groups of previously mentioned schemes. As shown in Table 3, when the metal elbow is not reinforced by CFRP, the deviation between theoretical calculation and FEA is less than $6 \%$ from Case 1 to Case 5. When the metal elbow is reinforced by CFRP with different thickness, along with increase in wall thickness of metal elbow and decrease in wall thickness of CFRP, the deviation is getting better from Case 5 to Case 12.

When the wall thickness of metal elbow reinforced by CFRP is $15 \mathrm{~mm}$ and $25 \mathrm{~mm}$, the deviation is smaller than $10 \%$. When the wall thickness of metal elbow $(9 \mathrm{~mm})$ is thin, the deviation is greater than $10 \%$. And along with 

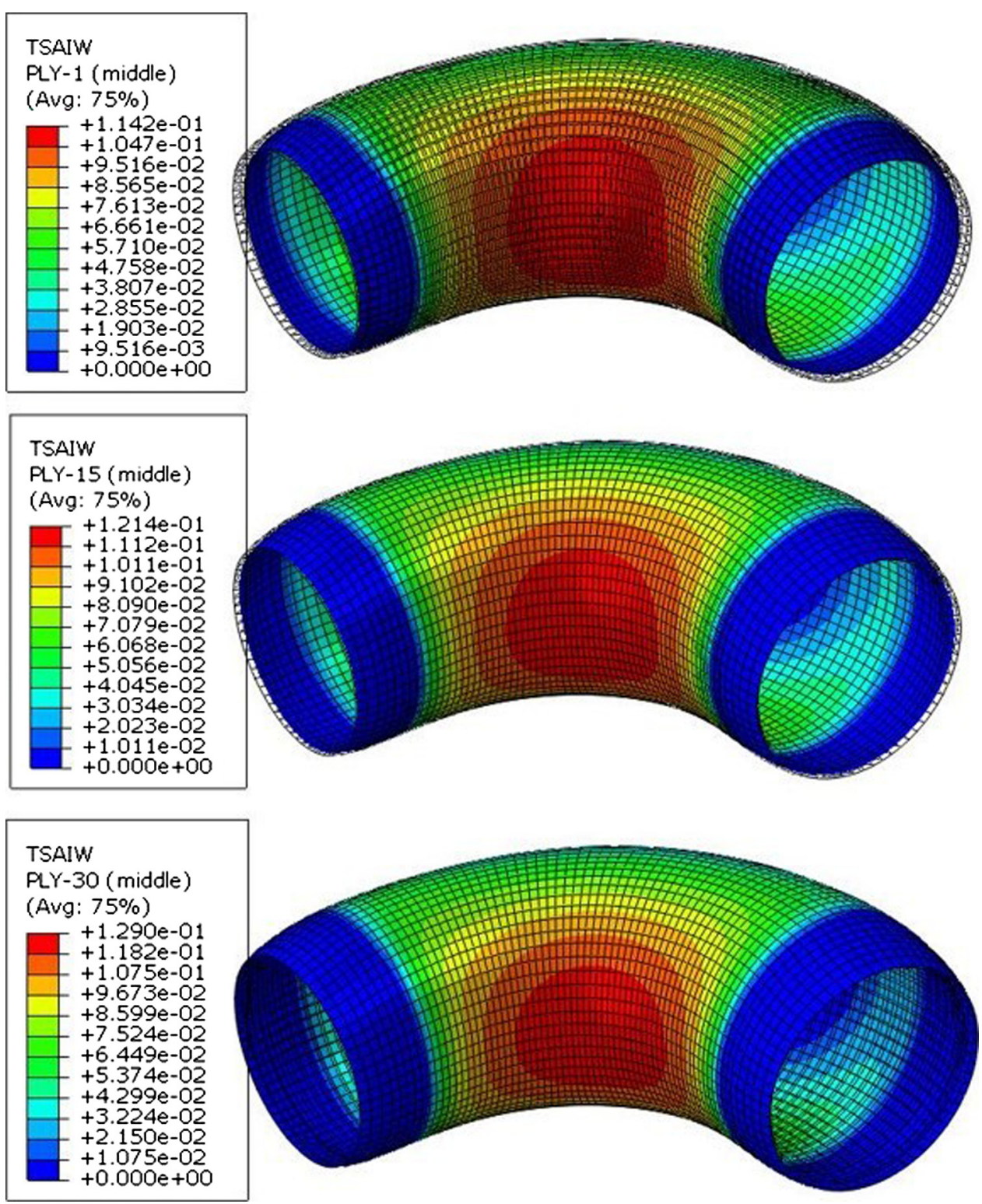

Fig. 7. the maximum Tsai-Wu failure indexes for ply-1, ply-15 and ply-30 are 0.114, 0.121 and 0.129 , respectively, all are less than 1 , indicating no failure occurred to all three plies.

increase in wall thickness of CFRP, the deviation is getting bigger. As can be concluded, the thicker the wall thickness of metal elbow, the thinner the wall thickness of CFRP, the better the accuracy of stress model will be.

\subsection{Failure analysis of metal elbow reinforced by CFRP}

For both the metal layer and CFRP layer of elbow, as indicated in Section 4.2.1, failure of metal layer has been determined based on whether the maximum circumferential stress carried by the metal layer of elbow is higher than the yield strength of the material. In the following, failure analysis on CFRP layer, of which failure is determined by using 3D Tsai-Wu criterion, will be unfolded.
Table 4 shows the Tsai-Wu failure index for each ply of CFRP regarding $9 \mathrm{~mm}$ metal elbow wrapped with $6 \mathrm{~mm}$ CFRP. According to this table, Tsai-Wu index ranged from 0.15 to 0.18 from ply 1 to 30 , all are smaller than 1 , indicating CFRP layer did not fail.

Table 5 shows the Tsai-Wu failure index for each ply of CFRP regarding $15 \mathrm{~mm}$ metal elbow wrapped with $6 \mathrm{~mm}$ CFRP. According to this table, Tsai-Wu index ranged from 0.11 to 0.13 from ply 1 to 30 , all are smaller than 1 , indicating CFRP layer did not fail.

Table 6 shows the Tsai-Wu failure index for each ply of CFRP regarding $25 \mathrm{~mm}$ metal elbow wrapped with $6 \mathrm{~mm}$ CFRP. According to this table, Tsai-Wu index ranged from 0.08 to 0.09 from ply 1 to 30 , all are smaller than 1 , indicating CFRP layer did not fail. 


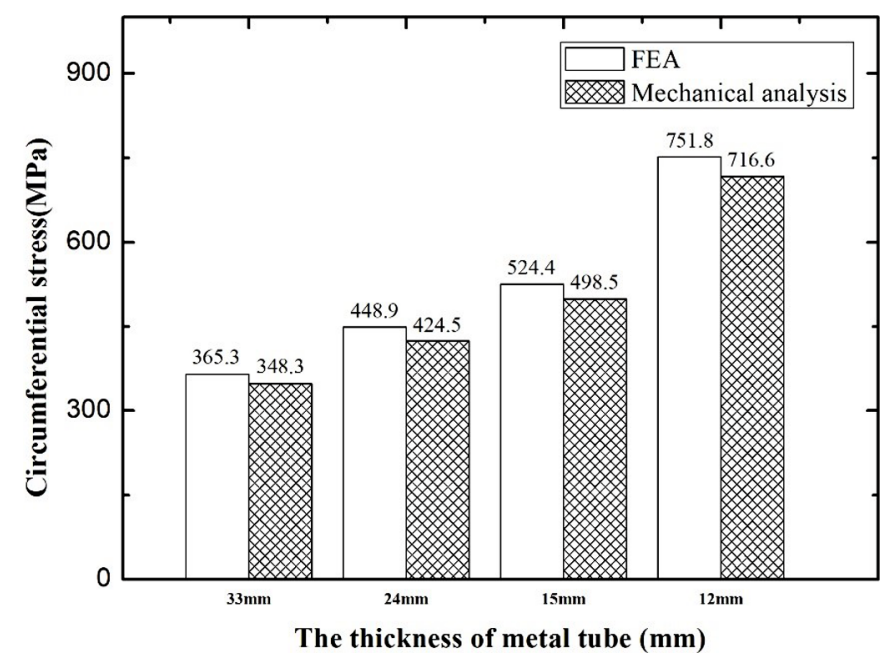

Fig. 8. Maximum circumferential stress of elbow with different wall thickness.

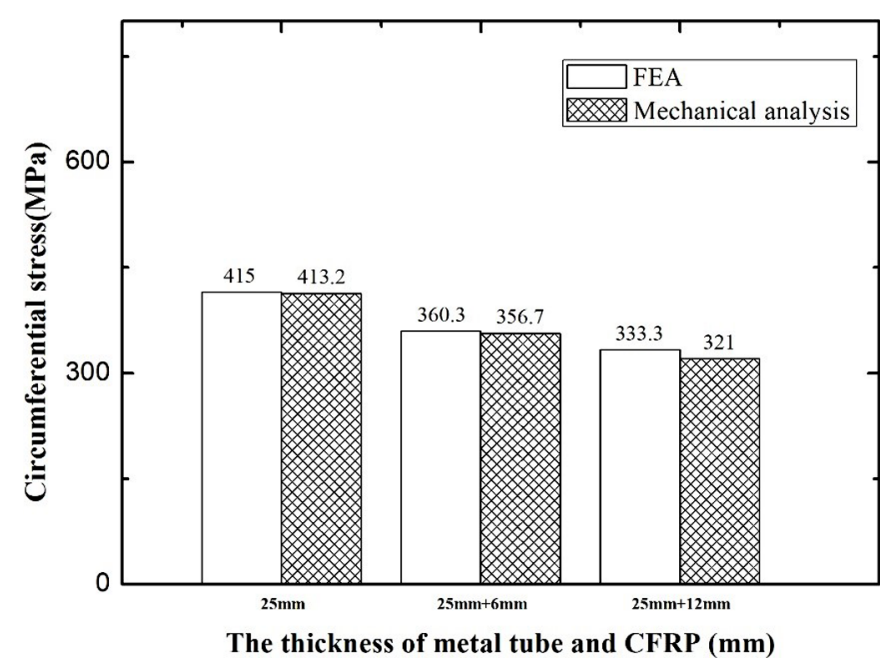

Fig. 9. Maximum circumferential stress of elbow reinforced by CFRP with different wall thickness (the wall thickness of metal elbow is $25 \mathrm{~mm}$ ).

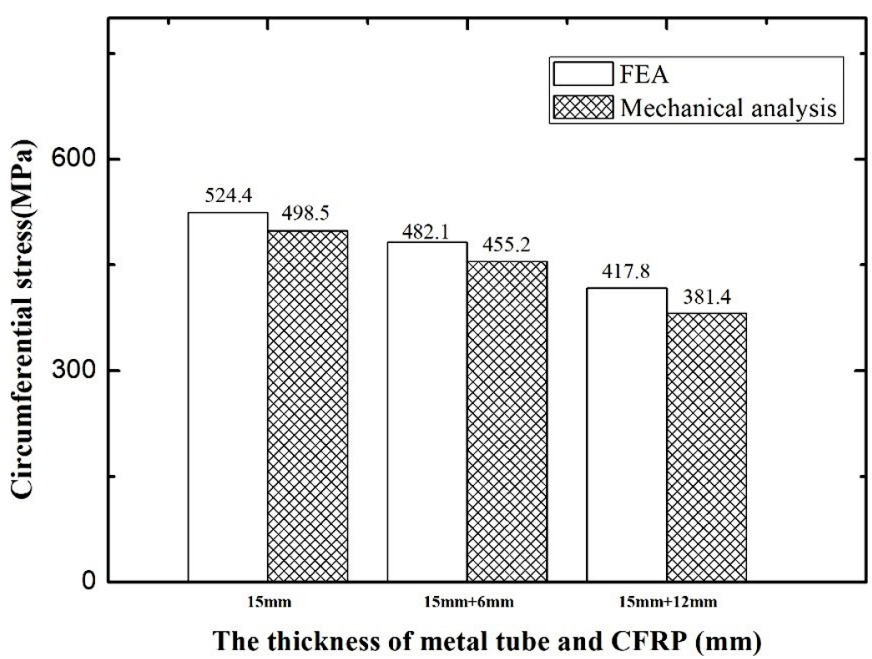

Fig. 10. Maximum circumferential stress of elbow reinforced CFRP with different wall thickness (the wall thickness of metal elbow is $15 \mathrm{~mm}$ ).

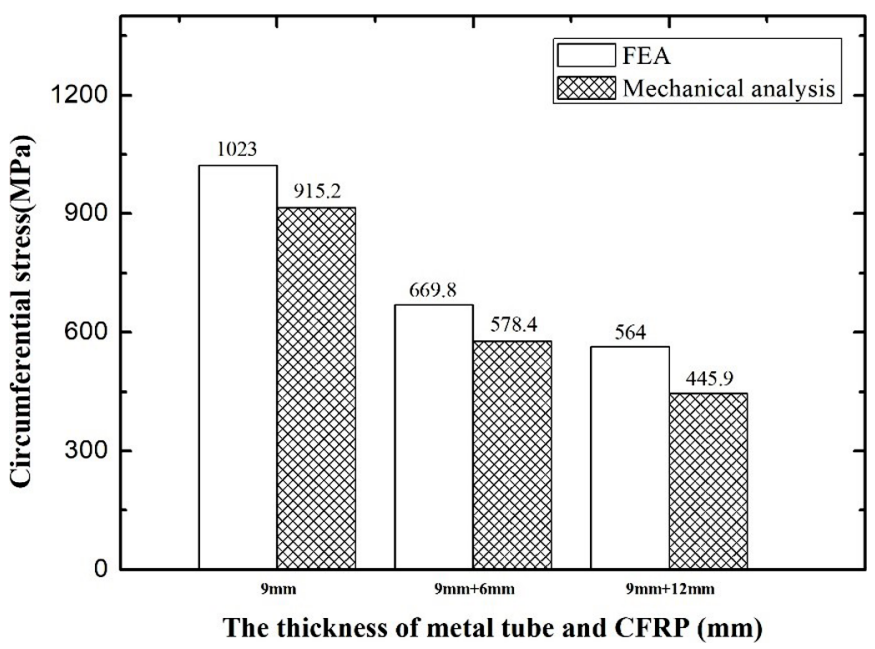

Fig. 11. Maximum circumferential stress of elbow reinforced by CFRP with different wall thickness (the wall thickness of metal elbow is $9 \mathrm{~mm}$ ).

Table 3. The deviation of theoretical calculation and FEA.

\begin{tabular}{|c|c|c|c|c|c|}
\hline Case & Composition of elbow & Deviation & Case & Composition of elbow & Deviation \\
\hline 1 & $33 \mathrm{~mm}$ Metal & $4.7 \%$ & 7 & $25 \mathrm{~mm}$ Metal $+12 \mathrm{~mm}$ CFRP & $3.7 \%$ \\
\hline 2 & $24 \mathrm{~mm}$ Metal & $5.4 \%$ & 8 & $15 \mathrm{~mm} \mathrm{Metal}+6 \mathrm{~mm} \mathrm{CFRP}$ & $3.6 \%$ \\
\hline 3 & $15 \mathrm{~mm}$ Metal & $4.9 \%$ & 9 & $15 \mathrm{~mm}$ Metal $+12 \mathrm{~mm}$ CFRP & $8.7 \%$ \\
\hline 4 & $12 \mathrm{~mm}$ Metal & $4.7 \%$ & 10 & $9 \mathrm{~mm}$ Metal & $10.5 \%$ \\
\hline 5 & $25 \mathrm{~mm}$ Metal & $0.4 \%$ & 11 & $9 \mathrm{~mm} \mathrm{Metal}+6 \mathrm{~mm} \mathrm{CFRP}$ & $13.6 \%$ \\
\hline 6 & $25 \mathrm{~mm} \mathrm{Metal}+6 \mathrm{~mm}$ CFRP & $1 \%$ & 12 & $9 \mathrm{~mm} \mathrm{Metal}+12 \mathrm{~mm} \mathrm{CFRP}$ & $20.9 \%$ \\
\hline
\end{tabular}


Table 4. Tsai-Wu failure index of every Ply for $9 \mathrm{~mm}$ metal elbow wrapped with $6 \mathrm{~mm}$ CFRP.

\begin{tabular}{llllllll}
\hline Ply No. & $\begin{array}{l}\text { Tsai-Wu } \\
\text { failure index }\end{array}$ & Ply No. & $\begin{array}{l}\text { Tsai-Wu } \\
\text { failure index }\end{array}$ & Ply No. & $\begin{array}{l}\text { Tsai-Wu } \\
\text { failure index }\end{array}$ & Ply No. & $\begin{array}{l}\text { Tsai-Wu } \\
\text { failure index }\end{array}$ \\
\hline 1st-ply & 0.1537 & 9th-ply & 0.16 & 17th-ply & 0.1661 & 25 th-ply & 0.1722 \\
2nd-ply & 0.1545 & 10th-ply & 0.1607 & 18th-ply & 0.1669 & 26 th-ply & 0.1729 \\
3rd-ply & 0.1553 & 11th-ply & 0.1615 & 19th-ply & 0.1676 & 27 th-ply & 0.1737 \\
4th-ply & 0.1561 & 12th-ply & 0.1623 & 20th-ply & 0.1684 & 28 th-ply & 0.1744 \\
5th-ply & 0.1569 & 13th-ply & 0.163 & 21th-ply & 0.1691 & 29 th-ply & 0.1752 \\
6th-ply & 0.1576 & 14th-ply & 0.1638 & 22th-ply & 0.1699 & 30th-ply & 0.1759 \\
7th-ply & 0.1584 & 15th-ply & 0.1646 & 23th-ply & 0.1706 & & \\
8th-ply & 0.1592 & 16th-ply & 0.1653 & 24th-ply & 0.1714 & & \\
\hline
\end{tabular}

Table 5. Tsai-Wu failure index of every Ply for $15 \mathrm{~mm}$ metal elbow wrapped with $6 \mathrm{~mm}$ CFRP.

\begin{tabular}{llllllll}
\hline Ply No. & $\begin{array}{l}\text { Tsai-Wu } \\
\text { failure index }\end{array}$ & Ply No. & $\begin{array}{l}\text { Tsai-Wu } \\
\text { failure index }\end{array}$ & Ply No. & $\begin{array}{l}\text { Tsai-Wu } \\
\text { failure index }\end{array}$ & Ply No. & $\begin{array}{l}\text { Tsai-Wu } \\
\text { failure index }\end{array}$ \\
\hline 1st-ply & 0.1142 & 9th-ply & 0.1183 & 17th-ply & 0.1224 & 25th-ply & 0.1264 \\
2nd-ply & 0.1147 & 10th-ply & 0.1188 & 18th-ply & 0.1229 & 26th-ply & 0.1269 \\
3rd-ply & 0.1152 & 11th-ply & 0.1193 & 19th-ply & 0.1234 & 27 th-ply & 0.1275 \\
4th-ply & 0.1157 & 12th-ply & 0.1198 & 20th-ply & 0.1239 & 28 th-ply & 0.128 \\
5th-ply & 0.1162 & 13th-ply & 0.1203 & 21th-ply & 0.1244 & 29 th-ply & 0.1285 \\
6th-ply & 0.1168 & 14th-ply & 0.1208 & 22th-ply & 0.1249 & 30 th-ply & 0.129 \\
7th-ply & 0.1173 & 15th-ply & 0.1214 & 23th-ply & 0.1254 & - & - \\
8th-ply & 0.1178 & 16th-ply & 0.1219 & 24th-ply & 0.1259 & - & - \\
\hline
\end{tabular}

Table 6. Tsai-Wu failure index of every Ply for $25 \mathrm{~mm}$ metal elbow wrapped with $6 \mathrm{~mm}$ CFRP.

\begin{tabular}{llllllll}
\hline Ply No. & $\begin{array}{l}\text { Tsai-Wu } \\
\text { failure index }\end{array}$ & Ply No. & $\begin{array}{l}\text { Tsai-Wu } \\
\text { failure index }\end{array}$ & Ply No. & $\begin{array}{l}\text { Tsai-Wu } \\
\text { failure index }\end{array}$ & Ply No. & $\begin{array}{c}\text { Tsai-Wu } \\
\text { failure index }\end{array}$ \\
\hline 1st-ply & 0.0813 & 9th-ply & 0.0835 & 17th-ply & 0.0856 & 25th-ply & 0.0878 \\
2nd-ply & 0.0815 & 10th-ply & 0.0837 & 18th-ply & 0.0859 & 26th-ply & 0.0881 \\
3rd-ply & 0.0818 & 11th-ply & 0.084 & 19th-ply & 0.0862 & 27 th-ply & 0.0884 \\
4th-ply & 0.0821 & 12th-ply & 0.0843 & 20th-ply & 0.0865 & 28 th-ply & 0.0886 \\
5th-ply & 0.0824 & 13th-ply & 0.0845 & 21th-ply & 0.0867 & 29th-ply & 0.0889 \\
6th-ply & 0.0826 & 14th-ply & 0.0848 & 22th-ply & 0.087 & 30th-ply & 0.0892 \\
7th-ply & 0.0829 & 15th-ply & 0.0851 & 23th-ply & 0.0873 & & \\
8th-ply & 0.0832 & 16th-ply & 0.0854 & 24th-ply & 0.0875 & & \\
\hline
\end{tabular}

It can be seen that CFRP layer do not fail when $6 \mathrm{~mm}$ CFRP is used to wrap around metal elbows with thickness of $9 \mathrm{~mm}, 15 \mathrm{~mm}$ and $25 \mathrm{~mm}$, and a conclusion can been drawn that when CFRP layer is increased to $12 \mathrm{~mm}$, it would not fail either. Therefore, failure of metal layer is the major cause for failure of elbow, i.e. if the metal layer fails, no matter whether the failure of the CFRP layer, the elbow reinforced by CFRP will be deemed in failure. This provides more proof to justify the accuracy and application of the stress model considering the effect of CFRP.

\section{Conclusions}

The stress model of thick-walled high pressure elbow reinforced by composite material has been discussed in this study. Based on the new stress model, finite element analyses are carried out to evaluate the stress model. The Failure analysis of metal elbow reinforced by CFRP has also been discussed. From the previously presented results, the following conclusion can be drawn as follows:

- Firstly, the maximum circumferential stress calculated by theoretical model is smaller than that of which 
calculated by FEA. Other factors may not be considered in the stress model;

- According to results of theoretical calculation and finite element simulation, the maximum circumferential stress is smaller than that of the elbow not being wrapped with CFRP. The thinner the wall thickness of metal elbow, the more obvious the effect of CFRP will be. When the wall thickness of metal elbow and CFRP is $9 \mathrm{~mm}$ and $12 \mathrm{~mm}$ respectively, comparing with the metal elbow with the same wall thickness, not being wrapped with CFRP, the maximum circumferential stress decreases by $48.1 \%$ averagely. In this case, the effect of CFRP is very obvious;

- When the metal elbow is reinforced by CFRP, within a given range of wall thickness, the thicker the wall thickness of metal elbow, the thinner the wall thickness of CFRP, the better the accuracy of stress model will be. When the wall thickness of metal elbow $(25 \mathrm{~mm})$ is thick, the deviation is smaller than $4 \%$. Therefore, the new stress model is an effective means for evaluating failure of high pressure elbow reinforced by composite material;

- It can be seen that CFRP layer do not fail when $6 \mathrm{~mm}$ CFRP is used to wrap around metal elbows with thickness of $9 \mathrm{~mm}, 15 \mathrm{~mm}$ and $25 \mathrm{~mm}$, and a conclusion can been drawn that when CFRP layer is increased to $12 \mathrm{~mm}$, it would not fail either. Therefore, failure of metal layer is the major cause for failure of elbow, i.e. if the metal layer fails, no matter whether the failure of the CFRP layer, the elbow reinforced by CFRP will be deemed in failure. This provides more proof to justify the accuracy and application of the stress model considering the effect of CFRP;

- In addition, the wrapping process of the elbow is also discussed in this study. Under the internal pressure, weak region of failure will occur firstly in the inner surface of the elbow, particularly on parts with the maximum curvature.

Acknowledgments. This work was supported by the National Natural Science Foundation of China-China Aerospace Science and Technology Corporation joint fund of aerospace advanced manufacturing technology research (No.U1537103) and the Fundamental Research Funds for the Central Universities (WUT:2017III047).

\section{References}

[1] H. Li, Y. Liang, H. Bao, CAM system for filament winding on elbows[J], J. Mater. Process. Technol. 161 (2005) 491-496

[2] H. Toutanji, S. Dempsey. Stress modeling of pipelines strengthened with advanced composites materials[J], ThinWalled Struct. 39 (2001) 153-165

[3] C. Anon, Robotised production of thermoplastic composites [J], Br. Plast. Rubber. 5 (1998) 30-31

[4] J.E. Creen, Overview of filament winding, SAMPE J. 37 (2001) 7-11

[5] J.M. Duell, J.M. Wilson, M.R. Kessler, Analysis of a carbon composite overwrap pipeline repair system [J], Int. J. Press. Vessels Pip. 85 (2008) 782-788

[6] S.W. Gong, K.Y. Lam, C. Lu, Structural analysis of a submarine pipeline subjected to underwater shock [J], Int. J. Press. Vessels Pip. 77 (2000) 417-423

[7] L.C.M. Meniconi, J.L.F. Freire, R.D. Vieira, J.L.C. Diniz, Stress analysis of pipelines with composite repairs[C], Int. Pipeline Conf. (2002) 2031-2037, DOI:10.1115/IPC200227372

[8] B.Y. Fang, A. Atrens, J.Q. Wang, E.H. Han, Z.Y. Zhu, W. $\mathrm{Ke}$, Review of stress corrosion cracking of pipeline steels in "low" and "high" pH solutions[J], J. Mater. Sci. 38 (2003) 127132

[9] T.M.B. Albarody, M.B. Zahiraniza, M.B. Taufiq, Stress analysis of reeled composite pipelines based on shallow shell theories[J]. Appl. Mech. Mater. 376 (2013) 181-184

[10] H. Altenbach, K. Naumenko, G. L'Vov, V. Sukiasov, A. Podgorny, Prediction of accumulation of technological stresses in a pipeline upon its repair by a composite band [J], Mech. Compos. Mater. 51 (2015) 139-156

[11] B. Pinheiro, I. Pasqualino, S. Cunha, Fatigue life assessment of damaged pipelines under cyclic internal pressure: Pipelines with longitudinal and transverse plain dents[J], Int. J. Fatigue 68 (2014) 38-47

[12] Y.J. Xie, W. Tang, Stress intensity factor for cracked submarine pipeline with concrete cover[J], Ocean Eng. 33 (2006) 1841-1852

[13] L.W. Wang, C.W. Du, Z.Y. Liu, X.H. Wang, X.G. Li, Influence of carbon on stress corrosion cracking of high strength pipeline steel[J], Corros. Sci. 76 (2013) 486-493

[14] B.Y. Yang, Engineering elastic-plastic mechanics [M], Tianjin University Press, Tianjin, 2003

[15] Z.X. Wang, Analysis and calculation of pipe stress [M], Water Conservancy and Electric Power Press, Beijing, 1983

Cite this article as: Y. Xiao, Y. Hu, J. Zhang, C. Song, X. Huang, Z. Liu, Stress analysis of metallic thick-walled high-pressure elbows overwrapped with composite material, Mechanics \& Industry 19, 204 (2018) 